\title{
Nursing Students' Willingness to Care for Older Adults
}

\author{
Insun Jang ${ }^{1}$, Younglee $\mathrm{Kim}^{2}$, Yeon $\mathrm{Kim}^{2}$ \\ ${ }^{1}$ Department of Nursing, Korean Bible University, Seoul, Republic of Korea \\ ${ }^{2}$ Department of Nursing, California State University San Bernardino, California, USA \\ Correspondence: Yeon Kim, Department of Nursing, California State University San Bernardino, 5500 University \\ Parkway, San Bernardino, CA 92407, USA.
}

Received: October 30, 2018

doi:10.11114/ijsss.v7i1.3726
Accepted: November 22, $2018 \quad$ Available online: December 21, 2018

URL: https://doi.org/10.11114/ijsss.v7i1.3726

\begin{abstract}
The older adult population has increased and is projected to grow. This population usually has chronic disorders that need continuous care. However, it has been reported nurses and nursing students have negative attitude towards older adults. This descriptive study aimed at investigating factors influencing nursing students' willingness to care for older adults. This study was conducted among 270 nursing students at the university in the United States from February 1 to February 28, 2017. Study participants answered the survey about quality and frequency of contact with older adults, anxiety about aging, empathy, attitude, and willingness to care for older adults. Tools in the study included; quality and frequency of contact with older adults; anxiety of aging scale; interpersonal reactivity index; attitude towards older adults; and willingness to care for older adults. The result showed factors influencing nursing students' willingness to care for older adults; the year (e.g. first, second, and third year) of nursing program, anxiety of aging, and empathy towards older adults. Therefore, this study recommends the nursing program be revised to improve nursing students' willingness to care for older adults by increasing empathy and quality of contact with older adults and decreasing anxiety about aging throughout the program.
\end{abstract}

Keywords: older adults, care, empathy, attitude, willing(ness), aging, anxiety

\section{Introduction}

The population of older adults currently continues to grow worldwide. In 2014, $14.5 \%$ of the United States population was 65 years old or older, and this proportion is predicted to increase to 23.5\% by 2060 (US Department of Commerce, 2015). Additionally, the risk of chronic disease is higher among older adults; in 2012, $60 \%$ of older adults were treated for at least two chronic diseases (Ward et al., 2012). Significantly, these chronic diseases require long-term care, and quality of nursing care is both essential and critical (Kim, 2011; Kim and Jeong, 2013).

Despite this growing trend, nurses often have negative attitude towards older adults because of insufficient knowledge about the aging process (Hanson, 2014). Moreover, the growing significance of older adults' health is illustrated by Shen and Xian (2012) who found that Chinese nursing students were reluctant to take care of older adults. Carlson (2013) also noted that Swedish nurses regarded caring for older adults as challenging, tedious, and depressing. Similarly, nursing students lacked knowledge about older adults and had negative perceptions towards them during the nursing program (Mandville-Anstey et al., 2014). Kydd et al. (2013) found negative perceptions of nursing students for older adult care barely improved between 1999 and 2009. Furthermore, nursing students were found to be uninterested in clinical practice related to older adult care (Kim et al., 2002; Kim, 2011; Kim and Jeong, 2013).

Zhang et al. (2016) argued that nursing students' positive attitudes and knowledge about older adults were critical to improving their willingness to care for older adults. Chi et al. (2016) also found that a positive attitude, an interest in the problems experienced by older adults, and volunteer activities were critical factors for nursing students' willingness to care for older adults. Other studies found that willingness to care for older adults was significantly associated with the attitude, experience, and knowledge provided during nursing education (Kwon et al., 2013), emotional intelligence and age (Kang and Sung, 2015), and ethical sensitivity (Hong et al., 2016).

However, there was a lack of studies investigating various factors relating to nursing students' willingness to care for older adults. Thus, Kim and Jang (2017) investigated nursing students' willingness to care for older adults at two universities in South Korea based on general characteristics, the quality and frequency of their contacts with older 
adults, and their aging-process anxiety, empathy, and attitude towards older adults. It was also found that there was no study considering these factors and investigating nursing students' willingness to care for older adults in the United States. Therefore, this present study modified the research design employed by Kim and Jang (2017), focused on the suggestions made by those authors for future studies, utilized the same variables, and collected data from nursing students in the States.

\section{Method}

\subsection{Research Design and Sample}

Our study used a descriptive cross-sectional design to investigate factors influencing the willingness of nursing students to care for older adults. A total of 270 nursing students participated in this study and they were sophomore, junior, and senior students from $X$ University that is a baccalaureate institution. The required sample size was 132 participants, as calculated using the $\mathrm{G}$ power program (version 3.1.3) for an alpha of .05 , a power of .80 , and an effect size of .30 . Accordingly, the sample size is appropriate.

\subsection{Instruments}

This study used the paper questionnaire containing five instruments to measure following concepts. Each instrument was validated in previous studies and some revision was made on instruments.

\subsubsection{Quality and Frequency of Contact with Older Adults}

Our study utilized contact measures developed by Hutchinson et al. (2010). This tool comprised six items scored on a 7-point Likert scale: three items each for the frequency and quality of contact with older adults, with higher scores indicating higher quality and frequency values. Contact frequency was measured using three items: "In the past, I have rarely interacted with elderly people" (reverse scored), "In the past I have interacted with elderly people in many areas of my life", and "The neighborhood(s) I grew up in had many elderly people". Contact quality was also measured using three items: "I have had many positive experiences with elderly people", "Over the course of my life I have had many elderly people as friends", and "In the past, my experiences with elderly people have been pleasant" (Hutchinson et al., 2010).

\subsubsection{Anxiety about Aging}

Aging anxiety was measured in this study using the Anxiety About Aging Scale (AAS) developed by Lasher and Faulkender (1993). The AAS included 20 items for assessing anxiety about aging in the following three subcategories with a 5-point Likert scale: fear of loss (11 items), fear of older adults (5 items), and psychological concerns (4 items). Higher scores indicate more fear of aging and loss, psychological concerns, and concerns about physical appearance.

Eleven items of fear of loss include "I fear it will be very hard for me to find contentment in old age", "I have never lied about my age in order to appear younger", "It doesn't bother me at all to imagine myself as being old", "I have never dreaded the day I would look in the mirror and see gray hair", "I have never dreaded looking old", "When I look in the mirror, it bothers me to see how my looks have changed with age", "I fear that when I am old all my friends will be gone", "The older I become, the more I worry about my health", "I get nervous when I think about someone else making decisions for me", "I worry that people will ignore me when I am old", and "I am afraid that there will be no meaning in life when I am old". Fear of older adults includes "I enjoy being around old people", "I like to go visit my older relatives", "I enjoy talking with old people", "I feel very comfortable when I am around an old person", and "I enjoy doing things for old people". Four Items of psychological concerns are "I will have plenty to occupy my time when I am old", "I expect to feel good about life when I am old", "I believe that I will still be able to do most things for myself when I am old", and finally "I expect to feel good about myself when I am old".

\subsubsection{Empathy towards Older Adults}

The Interpersonal Reactivity Index (IRI) instrument was developed by Davis (1980) to measure empathy toward older adults. Our study used only 20 items related to empathy out of 28 items with a content validity index (CVI) of .8 or above among three nursing faculty who were adult and gerontology content experts: 7 cognitive and 13 emotional empathy items. This tool was scored using a 5-point Likert scale, with higher scores showing greater empathy.

Seven cognitive empathy items are "I sometimes find it difficult to see things from the 'other guy's' point of view", "I try to look at everybody's side of a disagreement before I make a decision", "I sometimes try to understand my friends better by imagining how things look from their perspective", "If I'm sure I'm right about something, I don't waste much time listening to other people's arguments", "I believe that there are two sides to every question and try to look at them both", "When I'm upset at someone, I usually try to "put myself in his shoes' for a while", and "Before criticizing somebody, I try to imagine how I would feel if I were in their place". Thirteen emotional empathy items are "I often have tender and concerned feelings for old people less fortunate than me", "Sometimes I don't feel very sorry for old 
people when they are having problems", "When I see an old people being taken advantage of, I feel kind of protective towards them", "Old people's misfortunes do not usually disturb me a great deal", "When I see old people being treated unfairly, I sometimes don't feel very much pity for them", "I am often quite touched by things that I see happen", "In emergency situations, I feel apprehensive and ill-at-ease", "I sometimes feel helpless when I am in the middle of a very emotional situation", "When I see someone get hurt, I tend to remain calm", "Being in a tense emotional situation scares me", "I am usually pretty effective in dealing with emergencies", "I tend to lose control during emergencies", and "When I see someone who badly needs help in an emergency, I go to pieces".

\subsubsection{Attitude towards Older Adults}

Our study used the Semantic Differential Scale developed by Sanders et al. (1984) to measure the attitude of nursing students towards older adults. This tool consists of 20 items scored using a 7-point semantic differential scale to reflect a continuum between two opposite descriptive words of older adults, with lower scores indicating more positive attitudes. Twenty pairs of two opposite words are "Wise-Foolish", "Kind-Unkind", "Knowledgeable-Ignorant", "Happy-Sad", "Trustworthy-Untrustworthy", "Generous-Selfish", "Active-Inactive", "Neat-Untidy", "Friendly-Unfriendly", "Interesting-Boring", "Productive-Unproductive", "Healthy-Sick", "Good-Bad", "Flexible-Inflexible", "Independent-Dependent", "Liberal-Conservative", "Attractive-Unattractive", "Tolerant-Intolerant", "Optimistic-Pessimistic", and "Uncomplaining-Complaining".

\subsubsection{Willingness to Care for Older Adults}

The instrument developed by Zhang et al. (2016) for measuring willingness to care was used in this study to measure nursing students' willingness to care for older adults. This tool consisted of five items scored using a 5-point Likert scale, with higher scores indicating a higher willingness to care for older adults. These five items are "If given the chance, I am willing to care for the elderly", "No matter whether the elderly is my kinsfolk, I am still willing to take care for him/her", "Even when faced difficulties, I am willing to care for the elderly", "After graduating, I am willing to engage in aged care work after graduating", and "I am willing to take participation in long-term activities of elderly welfare".

\subsection{Procedure}

This study was approved by the Institutional Review Board (IRB) at X University (IRB-FY2017-70). Data were collected from February 1 to February 28, 2017 at X University. After the explanation of our study, informed consents were obtained from 270 nursing students and the 20-minute paper survey was self-administered. All participants completed the survey during class breaks and they represented the entire nursing students in the baccalaureate program of this university.

\subsection{Analysis}

IBM SPSS Statistics for Windows (version 21.0) was used to analyze the data in our study as follows:

1) Descriptive statistics was applied to nursing students' general characteristics, frequency and quality of contact with older adults, anxiety about aging processes, empathy for older adults, attitude towards older adults, and willingness to care for older adults.

2) ANOVA and $t$-test were used to analyze the relationship between nursing students' general characteristics and the frequency and quality of contact with older adults, anxiety about aging processes, empathy for older adults, attitude towards older adults, and willingness to care for older adults. Scheffe's test was used for multiple comparisons.

3) Pearson correlation coefficients were used to analyze correlations between the quality and frequency of contact, aging-process anxiety, empathy, attitude, and willingness to care for older adults among nursing students.

4) Multiple regression was used to investigate factors influencing willingness to care for older adults among nursing students.

\section{Results}

\subsection{Sample Characteristics}

This study involved 53 (19.6\%) male students and 217 (80.4\%) female students: 87 (32.2\%) second-year (sophomores), $92(34.1 \%)$ third-year (junior), and 91 (33.7\%) fourth-year (senior) students in nursing program. Hispanic students predominated $(n=107,39.6 \%)$, followed by Asians $(n=74,27.4 \%)$, Caucasians $(n=55,20.4 \%)$, and other ethnicities ( $n=34,12.6 \%$; including three African-Americans). There were $36(13.4 \%)$ students living in extended families and $232(86.6 \%)$ living in nuclear families. A minority of the participants had experience of older-adult-related volunteer activities $(n=106,39.4 \%)$. Most of the participants did not have older-adult-related nursing experience during their 
clinical practice $(n=209,77.4 \%)$, but took gerontology-related courses $(n=178,66.2 \%)$ (Table 1$)$.

Table 1. General characteristics $(n=270)$

\begin{tabular}{|c|c|c|c|}
\hline Variable & Categories & $n$ & $\%$ \\
\hline \multirow{2}{*}{ Gender } & Male & 53 & 19.6 \\
\hline & Female & 217 & 80.4 \\
\hline \multirow{3}{*}{ Year } & Second (Sophomore) & 87 & 32.2 \\
\hline & Third (Junior) & 92 & 34.1 \\
\hline & Fourth (Senior) & 91 & 33.7 \\
\hline \multirow{4}{*}{ Ethnicity* } & Caucasian & 55 & 20.4 \\
\hline & Hispanic & 107 & 39.6 \\
\hline & Asian & 74 & 27.4 \\
\hline & Other (including 3 African-Americans) & 34 & 12.6 \\
\hline \multirow{2}{*}{ Family type* } & Extended & 36 & 13.4 \\
\hline & Nuclear & 232 & 86.6 \\
\hline \multirow{2}{*}{ Experience of volunteer work* } & Yes & 106 & 39.4 \\
\hline & No & 163 & 60.6 \\
\hline \multirow{2}{*}{ Experience of older adult related nursing practice } & Yes & 209 & 77.4 \\
\hline & No & 61 & 22.6 \\
\hline \multirow{2}{*}{ Participation in older adult related courses* } & Yes & 178 & 66.2 \\
\hline & No & 91 & 33.8 \\
\hline
\end{tabular}

*Missing data excluded

\subsection{Descriptive Statistics in Variables}

The scores for the frequency and quality of contact with older adults were $4.83 \pm 1.20$ (mean \pm SD; range 1.00-7.00) and $4.94 \pm 1.13(1.00-7.00)$, respectively, both of which were right skew. The overall score for aging-process anxiety was

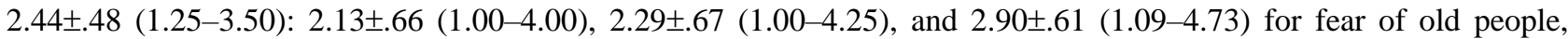
psychological concerns, and fear of loss, respectively. The overall score for empathy was 3.73土.37 (2.09-4.70):

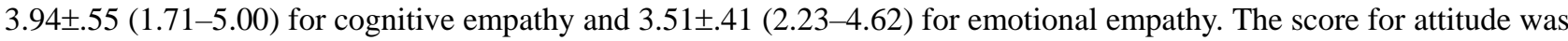
$3.36 \pm .71(1.00-6.55)$, while the score for willingness to care was $3.87 \pm .78(1.00-5.00)$, which was higher than the midpoint of the range (Table 2).

Table 2. Frequency and quality of contact, aging-process anxiety, empathy, attitude, and willingness to care for older adults $(\mathrm{n}=270)$

\begin{tabular}{lcccc}
\hline \multicolumn{1}{c}{ Variable } & $\begin{array}{c}\text { Value } \\
(\text { mean } \pm \text { SD) }\end{array}$ & $\begin{array}{c}\text { Value } \\
\text { (range) }\end{array}$ & $\begin{array}{c}\text { Score } \\
(\text { mean } \pm \text { SD) }\end{array}$ & $\begin{array}{c}\text { Score } \\
(\text { range) }\end{array}$ \\
\hline Contact Frequency & $14.52 \pm 3.61$ & $5.00-21.00$ & $4.83 \pm 1.20$ & $1.00-7.00$ \\
\hline Contact Quality & $14.82 \pm 3.39$ & $3.00-21.00$ & $4.94 \pm 1.13$ & $1.00-7.00$ \\
\hline Anxiety about Aging & $51.71 \pm 9.55$ & $22.00-85.00$ & $2.44 \pm 0.48$ & $1.25-3.50$ \\
$\quad$ Fear of old people & $10.65 \pm 3.28$ & $5.00-20.00$ & $2.13 \pm 0.66$ & $1.00-4.00$ \\
$\quad$ Psychological concerns & $9.15 \pm 2.67$ & $4.00-17.00$ & $2.29 \pm 0.67$ & $1.00-4.25$ \\
$\quad$ Fear of loss & $31.86 \pm 6.72$ & $12.00-52.00$ & $2.90 \pm 0.61$ & $1.09-4.73$ \\
\hline Empathy & $73.28 \pm 7.09$ & $44.00-93.00$ & $3.73 \pm 0.37$ & $2.09-4.70$ \\
$\quad$ Cognitive & $27.60 \pm 3.87$ & $12.00-35.00$ & $3.94 \pm 0.55$ & $1.71-5.00$ \\
$\quad$ Emotional & $45.65 \pm 5.32$ & $29.00-60.00$ & $3.51 \pm 0.41$ & $2.23-4.62$ \\
\hline Attitude & $67.29 \pm 14.16$ & $20.00-131.00$ & $3.36 \pm 0.71$ & $1.00-6.55$ \\
\hline Willingness to Care & $19.33 \pm 3.90$ & $5.00-25.00$ & $3.87 \pm 0.78$ & $1.00-5.00$ \\
\hline
\end{tabular}

\subsection{Analysis of Variance}

Findings indicated participants living in extended families had a significantly higher contact frequency with older adults $(\mathrm{t}=$ $2.490, \mathrm{p}=.013)$. Participants with the experience of older-adults-related voluntary work showed significantly higher frequency $(t=2.368, p=.019)$ and quality $(t=3.306, p=.001)$ of contact with older adults. They also showed significantly lower scores for aging-process anxiety $(\mathrm{t}=-2.240, \mathrm{p}=.026)$ and significantly higher scores for empathy towards older adults $(\mathrm{t}=1.986, \mathrm{p}=.048)$. The score for empathy was significantly higher for female participants than for males $(\mathrm{t}=2.517, \mathrm{p}$ $=.012$ ). There were significant differences among students in different years (second, third, and fourth years) of the nursing program in regard to their willingness to care for older adults, with second-year students exhibiting significantly higher willingness to care than the third- and fourth-year students $(\mathrm{t}=6.317, \mathrm{p}=.002)$. However, there was no significant difference in the attitude towards older adults regarding participants' general characteristics (Table 3). 
Table 3. General characteristics and differences in contact frequency and quality, aging process anxiety, empathy, attitude, and willingness to care for older adults according to general characteristics $(\mathrm{n}=270)$

\begin{tabular}{|c|c|c|c|c|c|c|c|c|c|c|c|c|c|}
\hline \multirow{2}{*}{ Variable } & \multirow{2}{*}{$\begin{array}{l}\text { Categor } \\
\mathrm{y}\end{array}$} & \multicolumn{2}{|l|}{$\begin{array}{l}\text { Contact } \\
\text { frequency }\end{array}$} & \multicolumn{2}{|c|}{ Contact quality } & \multicolumn{2}{|c|}{$\begin{array}{l}\text { Aging process } \\
\text { anxiety }\end{array}$} & \multicolumn{2}{|l|}{ Empathy } & \multicolumn{2}{|l|}{ Attitude } & \multicolumn{2}{|c|}{$\begin{array}{l}\text { Willingness to } \\
\text { Care }\end{array}$} \\
\hline & & $\begin{array}{l}\text { Mean } \pm S \\
\text { D }\end{array}$ & $\begin{array}{l}t / F \\
(p)\end{array}$ & $\begin{array}{l}\text { Mean } \pm S \\
\text { D }\end{array}$ & $\begin{array}{l}t / F \\
(p)\end{array}$ & $\begin{array}{l}\text { Mean } \pm S \\
\text { D }\end{array}$ & $\begin{array}{l}t / F \\
(p)\end{array}$ & $\begin{array}{l}\text { Mean } \pm S \\
\text { D }\end{array}$ & $\begin{array}{l}t / F \\
(p)\end{array}$ & $\begin{array}{l}\text { Mean } \pm S \\
\text { D }\end{array}$ & $\begin{array}{l}t / F \\
(p)\end{array}$ & $\begin{array}{l}\text { Mean } \pm S \\
\text { D }\end{array}$ & $\begin{array}{l}t / F \\
(p)\end{array}$ \\
\hline \multirow{3}{*}{ Year } & 2 nd & $\begin{array}{l}4.80 \pm 1 . \\
16\end{array}$ & \multirow{3}{*}{$\begin{array}{l}0.06 \\
3 \\
(.93 \\
9)\end{array}$} & $\begin{array}{l}4.98 \pm 0 . \\
99\end{array}$ & \multirow{3}{*}{$\begin{array}{l}0.11 \\
3 \\
(.89 \\
3)\end{array}$} & $\begin{array}{l}2.44 \pm 0 . \\
45\end{array}$ & \multirow{3}{*}{$\begin{array}{l}0.12 \\
8 \\
(.88 \\
0)\end{array}$} & $\begin{array}{l}3.67 \pm 0 . \\
35\end{array}$ & \multirow{3}{*}{$\begin{array}{l}1.68 \\
7 \\
(.18 \\
7)\end{array}$} & $\begin{array}{l}3.41 \pm 0 . \\
60\end{array}$ & \multirow{3}{*}{$\begin{array}{l}0.25 \\
0 \\
(.77 \\
9)\end{array}$} & $\begin{array}{l}4.07 \pm 0 . \\
70\end{array}$ & \multirow{3}{*}{$\begin{array}{l}6.31 \\
7 \\
(.00 \\
2)\end{array}$} \\
\hline & $3 \mathrm{rd}$ & $\begin{array}{l}4.84 \pm 1 . \\
25\end{array}$ & & $\begin{array}{l}4.91 \pm 1 . \\
21\end{array}$ & & $\begin{array}{l}2.46 \pm 0 . \\
47\end{array}$ & & $\begin{array}{l}3.76 \pm 0 . \\
33\end{array}$ & & $\begin{array}{l}3.34 \pm 0 . \\
87\end{array}$ & & $\begin{array}{l}3.88 \pm 0 . \\
85\end{array}$ & \\
\hline & 4th & $\begin{array}{l}4.87 \pm 1 \\
21\end{array}$ & & $\begin{array}{l}4.93 \pm 1 \\
18\end{array}$ & & $\begin{array}{l}2.43 \pm 0 . \\
51\end{array}$ & & $\begin{array}{l}3.76 \pm 0 . \\
42\end{array}$ & & $\begin{array}{l}3.35 \pm 0 . \\
62\end{array}$ & & $\begin{array}{l}3.66 \pm 0 . \\
73\end{array}$ & \\
\hline \multirow{2}{*}{ Gender } & Male & $\begin{array}{l}4.76 \pm 1 . \\
07\end{array}$ & \multirow{2}{*}{$\begin{array}{l}-\overline{0.52} \\
9 \\
(.59 \\
8)\end{array}$} & $\begin{array}{l}4.83 \pm 0 . \\
99\end{array}$ & \multirow{2}{*}{$\begin{array}{l}- \\
0.78 \\
5 \\
(.43 \\
3)\end{array}$} & $\begin{array}{l}2.52 \pm 0 . \\
47\end{array}$ & \multirow{2}{*}{$\begin{array}{l}1.31 \\
2 \\
(.19 \\
1)\end{array}$} & $\begin{array}{l}3.61 \pm 0 . \\
33\end{array}$ & \multirow{2}{*}{$\begin{array}{l}-2.51 \\
7 \\
(.01 \\
2)\end{array}$} & $\begin{array}{l}3.47 \pm 0 . \\
57\end{array}$ & \multirow{2}{*}{$\begin{array}{l}1.19 \\
2 \\
(.23 \\
4)\end{array}$} & $\begin{array}{l}3.74 \pm 0 . \\
81\end{array}$ & \multirow{2}{*}{$\begin{array}{l}- \\
1.28 \\
4 \\
(.20 \\
0)\end{array}$} \\
\hline & Female & $\begin{array}{l}4.86 \pm 1 \\
24\end{array}$ & & $\begin{array}{l}4.97 \pm 1 . \\
16\end{array}$ & & $\begin{array}{l}2.42 \pm 0 . \\
48\end{array}$ & & $\begin{array}{l}3.76 \pm 0 . \\
37\end{array}$ & & $\begin{array}{l}3.34 \pm 0 . \\
74\end{array}$ & & $\begin{array}{l}3.90 \pm 0 . \\
77\end{array}$ & \\
\hline \multirow{4}{*}{ Ethnicity } & $\begin{array}{l}\text { Caucasi } \\
\text { an }\end{array}$ & $\begin{array}{l}5.10 \pm 1 . \\
01\end{array}$ & \multirow{4}{*}{$\begin{array}{l}1.89 \\
2 \\
(.13 \\
1)\end{array}$} & $\begin{array}{l}5.16 \pm 1 . \\
04\end{array}$ & \multirow{4}{*}{$\begin{array}{l}2.27 \\
7 \\
(.08 \\
0)\end{array}$} & $\begin{array}{l}2.46 \pm 0 . \\
49\end{array}$ & \multirow{4}{*}{$\begin{array}{l}0.84 \\
0 \\
(.47 \\
3)\end{array}$} & $\begin{array}{l}3.70 \pm 0 . \\
34\end{array}$ & \multirow{4}{*}{$\begin{array}{l}0.57 \\
3 \\
(.63 \\
3)\end{array}$} & $\begin{array}{l}3.30 \pm 0 . \\
79\end{array}$ & \multirow{4}{*}{$\begin{array}{l}0.90 \\
6 \\
(.43 \\
9)\end{array}$} & $\begin{array}{l}3.69 \pm 0 . \\
90\end{array}$ & \multirow{4}{*}{$\begin{array}{l}1.67 \\
3 \\
(.17 \\
3)\end{array}$} \\
\hline & $\begin{array}{l}\text { Hispani } \\
\mathrm{c}\end{array}$ & $\begin{array}{l}4.65 \pm 1 . \\
23\end{array}$ & & $\begin{array}{l}5.03 \pm 1 . \\
13\end{array}$ & & $\begin{array}{l}2.43 \pm 0 . \\
46\end{array}$ & & $\begin{array}{l}3.73 \pm 0 . \\
36\end{array}$ & & $\begin{array}{l}3.31 \pm 0 . \\
69\end{array}$ & & $\begin{array}{l}3.91 \pm 0 . \\
73\end{array}$ & \\
\hline & Asian & $\begin{array}{l}4.93 \pm 1 \\
15\end{array}$ & & $\begin{array}{l}4.69 \pm 1 . \\
05\end{array}$ & & $\begin{array}{l}2.49 \pm 0 . \\
46\end{array}$ & & $\begin{array}{l}3.77 \pm 0 . \\
39\end{array}$ & & $\begin{array}{l}3.45 \pm 0 . \\
66\end{array}$ & & $\begin{array}{l}3.98 \pm 0 . \\
74\end{array}$ & \\
\hline & Other & $\begin{array}{l}4.83 \pm 1 \\
46\end{array}$ & & $\begin{array}{l}4.82 \pm 1 \\
35\end{array}$ & & $\begin{array}{l}2.34 \pm 0 . \\
57\end{array}$ & & $\begin{array}{l}3.69 \pm 0 . \\
43\end{array}$ & & $\begin{array}{l}3.45 \pm 0 . \\
73\end{array}$ & & $\begin{array}{l}3.78 \pm 0 . \\
78\end{array}$ & \\
\hline \multirow{2}{*}{$\begin{array}{l}\text { Family } \\
\text { type }\end{array}$} & $\begin{array}{l}\text { Extende } \\
\mathrm{d}\end{array}$ & $\begin{array}{l}5.30 \pm 1 . \\
30 \\
\end{array}$ & \multirow{2}{*}{$\begin{array}{l}2.49 \\
0 \\
(.01 \\
3)\end{array}$} & $\begin{array}{l}5.20 \pm 1 . \\
19\end{array}$ & \multirow{2}{*}{$\begin{array}{l}1.48 \\
9 \\
(.13 \\
8)\end{array}$} & $\begin{array}{l}2.33 \pm 0 . \\
63 \\
\end{array}$ & \multirow{2}{*}{$\begin{array}{l}-\overline{1} .17 \\
3 \\
(.24 \\
7)\end{array}$} & $\begin{array}{l}3.78 \pm 0 . \\
32\end{array}$ & $\begin{array}{l}0.84 \\
8\end{array}$ & $\begin{array}{l}3.39 \pm 0 . \\
72\end{array}$ & $\frac{0.24}{2}$ & $\begin{array}{l}3.75 \pm 0 . \\
84\end{array}$ & $\overline{0.96}$ \\
\hline & $\begin{array}{l}\text { Nuclear } \\
\text { Family }\end{array}$ & $\begin{array}{l}4.76 \pm 1 . \\
18\end{array}$ & & $\begin{array}{l}4.90 \pm 1 \\
12\end{array}$ & & $\begin{array}{l}2.46 \pm 0 . \\
45\end{array}$ & & $\begin{array}{l}3.72 \pm 0 . \\
38\end{array}$ & $\begin{array}{l}(.37 \\
9)\end{array}$ & $\begin{array}{l}3.36 \pm 0 . \\
71\end{array}$ & $\begin{array}{l}\text { 9) } \\
90\end{array}$ & $\begin{array}{l}3.88 \pm 0 \\
77\end{array}$ & $\begin{array}{l}5 \\
(.33 \\
5)\end{array}$ \\
\hline $\begin{array}{l}\text { Experienc } \\
\text { e of }\end{array}$ & Yes & $\begin{array}{l}5.06 \pm 1 . \\
22\end{array}$ & 2.36 & $\begin{array}{l}5.21 \pm 1 . \\
13\end{array}$ & $\begin{array}{l}3.30 \\
6\end{array}$ & $\begin{array}{l}2.36 \pm 0 . \\
52\end{array}$ & $\overline{2} .24$ & $\begin{array}{l}3.78 \pm 0 . \\
39 \\
\end{array}$ & $\begin{array}{l}1.98 \\
6\end{array}$ & $\begin{array}{l}3.29 \pm 0 . \\
69\end{array}$ & $\overline{1.32}$ & $\begin{array}{l}3.93 \pm 0 . \\
76\end{array}$ & 1.01 \\
\hline $\begin{array}{l}\text { volunteer } \\
\text { work }\end{array}$ & No & $\begin{array}{l}4.70 \pm 1 . \\
18\end{array}$ & $\begin{array}{l}\text { (.01 } \\
9)\end{array}$ & $\begin{array}{l}4.76 \pm 1 . \\
10\end{array}$ & $\begin{array}{l}\text { (.00 } \\
\text { 1) }\end{array}$ & $\begin{array}{l}2.50 \pm 0 . \\
44\end{array}$ & $\begin{array}{l}0 \\
(.02 \\
6)\end{array}$ & $\begin{array}{l}3.69 \pm 0 . \\
36\end{array}$ & $\begin{array}{l}(.04 \\
8)\end{array}$ & $\begin{array}{l}3.41 \pm 0 . \\
72\end{array}$ & $\begin{array}{l}7 \\
6) \\
6)\end{array}$ & $\begin{array}{l}3.83 \pm 0 \\
79\end{array}$ & $\begin{array}{l}(.30 \\
9)\end{array}$ \\
\hline $\begin{array}{l}\text { Older } \\
\text { adult }\end{array}$ & Yes & $\begin{array}{l}4.86 \pm 1 . \\
22\end{array}$ & 0.54 & $\begin{array}{l}4.94 \pm 1 . \\
17\end{array}$ & 0.04 & $\begin{array}{l}2.43 \pm 0 . \\
49\end{array}$ & $\overline{0} .78$ & $\begin{array}{l}3.75 \pm 0 . \\
38\end{array}$ & 1.91 & $\begin{array}{l}3.35 \pm 0 . \\
70\end{array}$ & $\overline{0} 62$ & $\begin{array}{l}3.83 \pm 0 . \\
79\end{array}$ & $\overline{1}$ \\
\hline $\begin{array}{l}\text { related } \\
\text { nursing } \\
\text { clinical } \\
\text { practice }\end{array}$ & No & $\begin{array}{l}4.77 \pm 1 . \\
14\end{array}$ & $\begin{array}{l}8 \\
4)\end{array}$ & $\begin{array}{l}4.93 \pm 1 . \\
00\end{array}$ & $\begin{array}{l}(.96 \\
8)\end{array}$ & $\begin{array}{l}2.48 \pm 0 \\
43\end{array}$ & $\begin{array}{l}4 \\
(.43 \\
4)\end{array}$ & $\begin{array}{l}3.65 \pm 0 . \\
34\end{array}$ & $\begin{array}{l}4 \\
\text { (.05 }\end{array}$ & $\begin{array}{l}3.41 \pm 0 . \\
73\end{array}$ & $\begin{array}{l}3 \\
. .53 \\
4)\end{array}$ & $\begin{array}{l}3.97 \pm 0 . \\
73\end{array}$ & $\begin{array}{l}2 \\
(.23 \\
8)\end{array}$ \\
\hline $\begin{array}{l}\text { Participati } \\
\text { on in older }\end{array}$ & Yes & $\begin{array}{l}4.90 \pm 1 . \\
20\end{array}$ & $3^{1.09}$ & $\begin{array}{l}4.97 \pm 1 . \\
12\end{array}$ & $\begin{array}{l}0.67 \\
4\end{array}$ & $\begin{array}{l}2.42 \pm 0 . \\
47\end{array}$ & $\overline{1.18}$ & $\begin{array}{l}3.73 \pm 0 . \\
37\end{array}$ & $\begin{array}{l}0.40 \\
7\end{array}$ & $\begin{array}{l}3.37 \pm 0 . \\
73\end{array}$ & $\begin{array}{l}0.25 \\
3\end{array}$ & $\begin{array}{l}3.89 \pm 0 . \\
78\end{array}$ & $\begin{array}{l}0.54 \\
3\end{array}$ \\
\hline $\begin{array}{l}\text { adult } \\
\text { related } \\
\text { courses }\end{array}$ & No & $\begin{array}{l}4.73 \pm 1 \\
22\end{array}$ & $\begin{array}{l}(.27 \\
5)\end{array}$ & $\begin{array}{l}4.87 \pm 1 . \\
15\end{array}$ & $\begin{array}{l}. .50 \\
1)\end{array}$ & $\begin{array}{l}2.49 \pm 0 . \\
49\end{array}$ & $\begin{array}{l}3 \\
(.23)\end{array}$ & $\begin{array}{l}3.71 \pm 0 . \\
38\end{array}$ & $\begin{array}{l}(.68 \\
5)\end{array}$ & $\begin{array}{l}3.35 \pm 0 . \\
67\end{array}$ & $\begin{array}{l}(.80 \\
0)\end{array}$ & $\begin{array}{l}3.83 \pm 0 . \\
78\end{array}$ & $\begin{array}{l}(.58 \\
8)\end{array}$ \\
\hline
\end{tabular}

\subsection{Correlations between Variables}

Findings indicated contact frequency was positively correlated with contact care quality $(\mathrm{r}=.496, \mathrm{p}<.001)$ and willingness to care $(\mathrm{r}=.042, \mathrm{p}=.039)$, whereas contact frequency was negatively correlated with aging-process anxiety ( $\mathrm{r}$ $=-.310, \mathrm{p}<.001)$ and attitude $(\mathrm{r}=-.186, \mathrm{p}=.002)$. Contact quality was also positively correlated with empathy $(\mathrm{r}=.156$, $\mathrm{p}=.012)$ and willingness to care $(\mathrm{r}=.248, \mathrm{p}<.001)$ while it was negatively correlated with anxiety of aging $(\mathrm{r}=-.546, \mathrm{p}$ $<.001)$ and attitude $(\mathrm{r}=-.367, \mathrm{p}<.001)$. Anxiety of aging was positively correlated with attitude $(\mathrm{r}=.300, \mathrm{p}<.001)$ but negatively correlated with empathy $(\mathrm{r}=-.176, \mathrm{p}=.004)$ and willingness to care $(\mathrm{r}=-.253, \mathrm{p}<.001)$. Empathy was positively correlated with willingness to care $(r=.163, p=.008)$ but negatively correlated with attitude $(r=-.141, p$ $=.024)$. Attitude was negatively correlated with willingness to care $(\mathrm{r}=-.213, \mathrm{p}<.001)$ (Table 4$)$. 
Table 4. Correlations between contact frequency and quality, aging-process anxiety, empathy, attitude, and willingness to care for older adults $(\mathrm{n}=270)$

\begin{tabular}{|c|c|c|c|c|c|c|}
\hline & $\begin{array}{c}\text { Contact frequency } \\
r(p)\end{array}$ & $\begin{array}{l}\text { Contact } \\
\text { quality } \\
r(p)\end{array}$ & $\begin{array}{c}\text { Anxiety about } \\
\text { Aging } \\
r(p)\end{array}$ & $\begin{array}{l}\text { Empathy } \\
\qquad r(p)\end{array}$ & $\begin{array}{l}\text { Attitude } \\
\qquad r(p)\end{array}$ & $\begin{array}{c}\text { Willingness to } \\
\text { Care } \\
r(p)\end{array}$ \\
\hline Contact frequency & 1.00 & & & & & \\
\hline Contact quality & $\begin{array}{c}.496 \\
(<.001)\end{array}$ & 1.00 & & & & \\
\hline $\begin{array}{l}\text { Anxiety about } \\
\text { Aging }\end{array}$ & $\begin{array}{l}-.310 \\
(<.001)\end{array}$ & $\begin{array}{l}-.546 \\
(<.001)\end{array}$ & 1.00 & & & \\
\hline Empathy & $\begin{array}{l}.042 \\
(.495)\end{array}$ & $\begin{array}{l}.156 \\
(.012)\end{array}$ & $\begin{array}{l}-.176 \\
(.004)\end{array}$ & 1.00 & & \\
\hline Attitude & $\begin{array}{l}-.186 \\
(.002)\end{array}$ & $\begin{array}{l}-.367 \\
(<.001)\end{array}$ & $\begin{array}{c}.300 \\
(<.001)\end{array}$ & $\begin{array}{l}-.141 \\
(.024)\end{array}$ & 1.00 & \\
\hline Willingness to Care & $\begin{array}{l}.126 \\
(.039)\end{array}$ & $\begin{array}{c}.248 \\
(<.001)\end{array}$ & $\begin{array}{l}-.253 \\
(<.001)\end{array}$ & $\begin{array}{l}.163 \\
(.008)\end{array}$ & $\begin{array}{l}-.213 \\
(<.001)\end{array}$ & 1.00 \\
\hline
\end{tabular}

3.5 Factors Influencing the Willingness to Care

Multiple regression was used to analyze how the year of the participants affected their willingness to care, contact frequency, contact quality, attitude, empathy, and anxiety of aging. A test of multicollinearity between independent variables applied before a multiple-regression test revealed no multicollinearity: ranges of tolerance and variation inflation factor were .542-.978 and 1.023-1.843, respectively. The Durbin-Watson statistic was 1.941, indicating no autocorrelation between the variables. There was statistically significant multiple regression $(\mathrm{F}=6.844, \mathrm{p}<.001)$. The value of $R^{2}$ was .12 , indicating approximately $12 \%$ of the variance of willingness to care for older adults could be estimated by year, empathy, and anxiety of aging (Table 5). The most important factor influencing willingness to care for older adults was the year of the nursing program $(\beta=.178, p=.003)$, followed by anxiety of aging $(\beta=-.140, p=.049)$ and empathy towards older adults $(\beta=.131, \mathrm{p}=.031)$.

Table 5. Factors influencing willingness to care for older adults $(n=270)$

\begin{tabular}{|c|c|c|c|c|c|}
\hline \multirow{2}{*}{ Variable } & \multicolumn{5}{|c|}{ Care willingness } \\
\hline & $\mathrm{B}$ & SE & $\beta$ & $t$ & $p$ \\
\hline (Constant) & 3.337 & .760 & & 4.391 & $<.001$ \\
\hline Year $(1=$ Second $)$ & 0.301 & .100 & .178 & 3.002 & .003 \\
\hline Contact frequency & 0.008 & .044 & .012 & .180 & .857 \\
\hline Contact quality & 0.072 & .055 & .104 & 1.302 & .194 \\
\hline Attitude & -0.134 & .071 & -.120 & -1.880 & .061 \\
\hline Empathy & 0.281 & .130 & .131 & 2.163 & .031 \\
\hline Anxiety about Aging & -0.232 & .117 & -.140 & -1.976 & .049 \\
\hline
\end{tabular}

Adj. $R^{2}=.121, F=6.844, p<.001$

Durbin-Watson statistic $=1.941$

\section{Discussion}

This is a replication study of one first performed by Kim and Jang (2017), so this discussion is focused to compare these two studies. Significantly, the frequency and quality of contact with older adults were higher than those of college students with non-nursing majors in the study by Kim (2013) and those of Korean nursing students in the study by Kim and Jang (2017). The mean score for aging-process anxiety was lower than that of college students with non-nursing majors (Kim, 2013) and Korean nursing students (Kim and Jang, 2017). Among subgroups of aging-process anxiety, the participants in the present study showed much lower scores for the fear of old people and psychological concerns than Korean nursing students (Kim and Jang, 2017), whereas the mean score for the fear of loss was only slightly lower in this study.

Also, nursing students in the present study showed the highest score for the fear of loss, followed by psychological concerns and then fear of older adults. Conversely, Korean nursing students in the study by Kim and Jang (2017) showed more fear of older people than psychological concerns. Interestingly, the score for empathy towards older adults in this study - including both cognitive and emotional empathy — was higher than that of Korean nursing students, and cognitive empathy was greater than emotional empathy in both studies. Further, the score for the attitude towards older adults was lower than the middle range, while previous studies found the attitudes of nurses and nursing students were within the middle range (Kim et al., 2002; Kim, 2011; Kim, and Jeong, 2013; Kim et al., 2016; Kim and Jang, 2017; Kwon et al., 2013). 
This study found second-year nursing students (sophomores) exhibited the highest score for willingness to care for older adults, whereas Kim and Jang (2017) revealed that senior students had the highest score. The factors influencing willingness to care for older adults in this study were the year of the nursing program, empathy, and anxiety about aging, which indicated approximately $12 \%$ of the variance of willingness to care for older adults was estimated by these factors. On the other hand, Kim and Jang (2017) reported senior students, living in an extended family, and participation in gerontology-related nursing were associated with willingness to care, with $40.3 \%$ of the variance of willingness to care being estimated by these factors.

The year of the nursing program (e.g. sophomore, junior, and senior) was the most important factor influencing willingness to care for older adults. This study showed sophomores exhibited the highest willingness to care while Kim and Jang (2017) found senior students had the highest willingness. The study by Jackson et al. (2017) also showed nursing students had more interest and confidence for older adult care toward completing their program, which means senior students had the higher interest and confidence than sophomore or junior students did. This suggests nursing students may have lost interest in caring older adults, are more interested in other fields of nursing, or are not confident enough to care for older adults. Therefore, clinical courses of the current nursing program may need to be revised to encourage students to develop willingness to care for older adults as they proceed through a nursing program, and to ensure qualified nurses will fulfill the needs for older adult care in aging societies. For example, assigning an older adult more often to a student may help the student experience with older adults in the clinical setting. Also, clinical faculty need to positively influence students about caring older adults during the clinical rotation, which can help students learn geriatric nursing care without being biased on older adults.

Anxiety of aging was reported as the second most important factor influencing the willingness to care for older adults in this study. Interestingly, anxiety was significantly lower among students who did volunteer work for older adults, had high frequency and quality of contact with older adults, and had empathy and positive attitudes towards older adults. These findings were similar to those of Hutchison et al. (2010), Kim (2013), and Kim and Jang (2017). However, Kim and Jang (2017) showed living in an extended family and having experience with older-adult-related nursing (rather than volunteer work) were related to lower anxiety about aging. Thus, when students understand aging as a natural process through their contacts with older adults, they might be less afraid of aging, which could increase their willingness to care for older adults. Accordingly, causes of anxiety about aging among students need to be identified and reduced. Therefore, the nursing program should be redesigned to motivate students to participate in older-adults-related volunteer activities to enhance the frequency and quality of contact with older adults, with an emphasis on empathy and positive attitudes throughout the program. For instance, curricula of the program may need to include volunteer activities of providing health care services specifically designed for older adult population to the community so that students can participate in the activity to develop willingness to care for that population.

Empathy towards older adults was another factor influencing the willingness to care for older adults among nursing students. Empathy was associated with the contact quality, anxiety of aging, and attitude for older adults. Accordingly, empathy needs to be developed by increasing contact quality with older adults and improving attitudes towards older adults throughout the nursing program and relevant activities. Considering senior nursing students-who had more clinical experiences with older adults than second-year students - had lower willingness to care for older adults, the quality of contact with older adults is more important than the contact frequency, which should be improved during clinical rotations involving older adult patients. Therefore, nursing program should help students more focus on older adults' psychological aspect as well as physical aspect so that they can develop empathy towards older adults.

This study is significant in that it examined the factors influencing the willingness of nursing students to care for older adults, not just their attitudes or behaviors towards them. The age of societies has raised the need for qualified nurses in taking care of older adults, and the findings of this study suggest nursing programs needs to be modified to meet these needs, by increasing the frequency and quality of contact with older adults, lowering anxiety of aging, increasing positive attitudes, and developing empathy. Such approaches would enhance the nursing students' willingness to care for older adults by ensuring they accurately understand aging and relevant health problems without false concepts or prejudice regarding older adults.

This study has some limitations. It is difficult to generalize findings of the study because it was conducted among baccalaureate undergraduate nursing students at a local university in a single state. Thus, future studies with a similar research design in different colleges and programs are recommended, including vocational and associate-degree nursing programs. Also, nurses' interaction with older adult patients and its influence on nursing students need to be investigated in the future study because students often observe nurses' care for patients during the clinical rotation. Another suggestion is to evaluate nursing students' willingness to care for older adults after performing revised nursing programs with the aim of increasing their willingness to care for older adults. 


\section{Conclusion}

Nursing students' willingness to care for older adults demonstrated differently based on their empathy for older adults, anxiety level of aging, and school year in this study. This indicates that nursing educators should consider these areas when planning nursing courses, especially relevant to geriatric care. The growing older adult population in societies has correspondingly increased the need for qualified nurses to care for them. Additionally, it is one of the critical responsibilities for nurses to maintain and improve societal health as health care professionals. Thus, a high willingness to care for older adults needs to be engendered in nursing students - that is, our future nurses. Therefore, nursing education should include improved and various curricular contents and methods for older adult care to meet this societal need.

\section{References}

Carlson, E. (2013). Meaningful and enjoyable or boring and depressing? The reasons student nurses give for and against a career in aged care. Journal of Clinical Nursing, 24, 602-604. https://doi.org/10.1111/jocn.12425

Chi, M. J., Shyu, M. L., Wang, S. Y., Chuang, H. C., \& Chuang, Y. H. (2016). Nursing students' willingness to care for older adults in Taiwan. Journal of Nursing Scholarship, 48(2), 172-178. https://doi.org/10.1111/jnu.12197

Davis, M. H. (1980). A multidimensional approach to individual differences in empathy. JSAS Catalog of Selected Documents in Psychology, 10, 85.

Hanson, R. M. (2014). Is elderly care affected by nurse attitudes? A systematic review. British Journal of Nursing, 23(4), 225-229. https://doi.org/10.12968/bjon.2014.23.4.225

Hong, S. H., Park, Y. H., \& Moon, J. S. (2016). Relationship between moral sensitivity and elderly nursing practice of nurses. Journal of the Korean Bioethics Association, 17(1), 75-87.

Hutchinson, P., Fox, E., Laas, A. M., Matharu, J., \& Urzi, S. (2010). Anxiety, outcome expectancies, and young people's willingness to engage in contact with the elderly. Educational Gerontology, 36, 1008-1021. https://doi.org/10.1080/03601271003723586

Jackson, J., Clark, A., Pearse, L., Miller, E. A., Stanfield, H., \& Cunningham, C. L. Jr. (2017). Explaining student interest and confidence in providing care for older adults: The role of demographics and life experiences. Journal of Gerontological Nursing, 43(3), 13-18. https://doi.org/10.3928/00989134-20160901-04

Kang, H. S., \& Sung, K. W. (2015). Influence of emotional intelligence and organizational commitment on geriatric nursing practice of nurses in long-term care hospitals. Journal of the Korean Gerontological Nursing, 17(1), 29-37. https://doi.org/10.17079/jkgn.2015.17.1.29

Kim, J. H., \& Jeong, H. S. (2013). Influencing factor on the attitudes toward elders among nurses working at geriatric hospitals: Focusing on Empathy. Journal of the Korea Contents Association, 13(12), 840-850. https://doi.org/10.5392/JKCA.2013.13.12.840

Kim, M. H. (2011). Nursing students' knowledge, attitude and behavior toward elderly. The Korean Journal of Health Service Management, 5(4), 113-126. https://doi.org/10.12811/kshsm.2011.5.4.113

Kim, S. O., Oh, H. S., \& Wang, M. S. (2016). A study on the nursing students' knowledge, attitudes and behavior towards the elderly. Journal of Korean Academy of Society Nursing Education, 22(2), 163-170. https://doi.org/10.5977/jkasne.2016.22.2.163

Kim, S. Y., Yang, K. M., Oh, H. E., \& Kim, J. S. (2002). Nursing and social welfare students' knowledge and attitudes toward the elderly. Journal of the Korea Gerontological Society, 22(3), 21-36.

Kim, Y. S., \& Jang, I. (2017). The factors associated with care willingness towards older adults among nursing students. Journal of the Korean Data Analysis Society, 19(3), 1669-1683.

Kwon, Y. H., Lee, H. Y., \& Hwang, S. S. (2013). A study on the knowledge, attitude and nursing practice of the nurses-towards the elderly in geriatric hospitals. Journal of the Korea Academia-Industrial Cooperation Society, 14(11), 5785-5791. https://doi.org/10.5762/KAIS.2013.14.11.5785

Kydd, A., Wild, D., \& Nelson, S. (2013). Attitudes towards caring for older people: Findings and recommendations for practice. Nursing Older People, 25(4), 21-28. https://doi.org/10.7748/nop2013.05.25.4.21.e769Part2

Lasher, K. P., \& Faulkender, P. J. (1993). Measurement of aging anxiety: Development of anxiety about aging scale. International Journal of Aging and human development, 37(4), 247-259. https://doi.org/10.2190/1U69-9AU2-V6LH-9Y1L

Mandville-Anstey, S., Ward, P., Grainger, P., \& Foley, V. (2014). Perspectives. The Journal of the Gerontological Nursing 
Association, 37(2), 6-14.

Sanders, G. F., Montgomery, J. E., Pittman, Jr. J. F., \& Balkwell, C. (1984). Youth's attitudes toward the elderly. Journal of Applied Gerontology, 3(1), 59-70. https://doi.org/10.1177/073346488400300107

US Department of Commerce (2015). Projections of the size and composition of the U.S. population 2014 to 2060 : Current population reports. Retrieved from https://www.census.gov/content/dam/Census/library/publications/2015/demo/p25-1143.pdf

Ward, B. W., Schiller, J. S., \& Goodman, R. A. (2012). Multiple chronic conditions among US Adults: A 2012 update. Washington, DC: U.S. Government Printing Office.

Zhang, S., Liu, Y. H., Zhang, H. F., Meng, L. N., \& Liu, P. X. (2016). Determinants of undergraduate nursing students' care willingness towards the elderly in China: Attitudes, gratitude and knowledge. Nurse Education Today, 43, $28-33$. https://doi.org/10.1016/j.nedt.2016.04.021

\section{Copyrights}

Copyright for this article is retained by the author(s), with first publication rights granted to the journal.

This is an open-access article distributed under the terms and conditions of the Creative Commons Attribution license which permits unrestricted use, distribution, and reproduction in any medium, provided the original work is properly cited. 